\title{
Investigation of Circularly Polarized Loop Antennas With a Parasitic Element for Bandwidth Enhancement
}

\author{
RongLin Li, Senior Member, IEEE, Gerald DeJean, Student Member, IEEE, Joy Laskar, Fellow, IEEE, and \\ Manos M. Tentzeris, Senior Member, IEEE
}

\begin{abstract}
It is demonstrated that the bandwidth of circular polarization (CP) can be significantly increased when one more parasitic loop is added inside the original loop. A single-loop antenna has only one minimum axial ratio (AR) point while the two-loop antenna can create two minimum AR points. An appropriate combination of the two minimum AR points results in a significant enhancement for the CP bandwidth. A comprehensive study of the new type of broad-band circularly polarized antennas is presented. Several loop configurations, including a circular loop, a rhombic loop, and a dual rhombic loop with a series feed and a parallel feed, are investigated. The $A R(\leq 2 \mathrm{~dB})$ bandwidth of the circular-loop antenna with a parasitic circular loop is found to be $20 \%$, more than three times the AR bandwidth of a single loop. For the rhombic-loop antenna with a parasitic rhombic loop, an AR bandwidth ( $A R \leq 2 \mathrm{~dB}$ ) of more than $40 \%$ can be achieved by changing the rhombus vertex angle. The $A R(\leq 2 \mathrm{~dB})$ bandwidths of the series-fed and parallel-fed dual rhombic-loop antennas with a parasitic element are $30 \%$ and $50 \%$, respectively. A broad-band balun is incorporated into the series-fed dual rhombic-loop antenna for impedance matching. The broad-band CP performance of the loop antennas is verified by experimental results.
\end{abstract}

Index Terms-Broad-band antenna, circularly polarized (CP) antenna, loop antenna.

\section{INTRODUCTION}

$\mathbf{L}$ OOP antennas (such as circular, square, triangular, rectangular, and rhombic loops) are usually used as linearly polarized antennas [1]. In recent years, it has been found that a loop antenna can also radiate circularly polarized (CP) waves if a gap is introduced on the loop [2]-[5]. The reason for the CP radiation is the traveling-wave current distribution that gets excited along the loop. Compared to conventional center-fed planar spiral antennas [6], the CP loop antennas have two important advantages. First, the loop antenna is fed on its perimeter, making it possible to directly integrate an antenna array in a coplanar stripline circuit [7], [8]. Second, the sense of the circular polarization can be easily switched from left-hand to right-hand, and vice versa, by altering the gap positions using radio-frequency (RF) switches such as microelectro-mechanical systems (MEMS) [9], optoelectronic switches [10], or PIN diodes [11]. Even though it is possible for some loop configurations (such as rhombic loops)

Manuscript received January 14, 2005; revised August 12, 2005. The authors wish to acknowledge the support of Georgia Electronic Design Center (GEDC), the NSF CAREER Award ECS-9984761, the NSF Grant ECS-0313951, and the NSF Packaging Research Center.

The authors are with the Georgia Electronic Design Center, School of Electrical and Computer Engineering, Georgia Institute of Technology, Atlanta, GA 30332-0250 USA (e-mail: rlli@ece.gatech.edu).

Digital Object Identifier 10.1109/TAP.2005.859917 to achieve a bandwidth of more than $20 \%$ for an axial ratio (AR) of less than $2 \mathrm{~dB}$, the bandwidth of the CP loop antennas is still much less than that achieved by spiral antennas [12]. In this paper, it will be demonstrated that the AR bandwidth of a $\mathrm{CP}$ loop antenna can be significantly increased by adding a similar loop (also with a gap, called "parasitic element" in general) inside the original loop. The addition of a parasitic element can produce two minimum AR points that (by an appropriate combination) lead to a considerable enhancement for the AR bandwidth. Since the additional parasitic element is placed inside the original loop and there is no direct electrical connection to its surrounding, there is no significant increase in the size and complexity of the antenna structure. In this paper, several loop configurations will be investigated, including a circular loop, a rhombic loop, a series-fed dual rhombic loop, and a parallel-fed dual rhombic loop. It will be shown that for a circular-loop antenna the $\mathrm{AR}(\leq 2 \mathrm{~dB})$ bandwidth be can be increased from $6.5 \%$ to $20 \%$ by the addition of a parasitic circular loop. For a rhombic loop antenna with a parasitic element, it is possible to achieve an $\mathrm{AR}(\leq 2 \mathrm{~dB})$ bandwidth of more than $40 \%$ by adjusting the rhombus vertex angle. For the series-fed and parallel-fed dual rhombic-loop antenna with a parasitic loop, the bandwidths for $\mathrm{AR} \leq 2 \mathrm{~dB}$ of $30 \%$ and $50 \%$ can be obtained, respectively.

First the bandwidth improvement of a circular-loop antenna with a parasitic element will be demonstrated. Then the single rhombic and dual rhombic loops with a parasitic element will be investigated in order to further increase the AR bandwidth of loop antennas. Finally, two experimental examples (a circular loop and a series-fed dual rhombic loop with broad-band balun) will be presented to verify the theoretical and simulation results.

\section{Circular-Loop Antenna With a Parasitic Element}

\section{A. Antenna Structure}

As shown in Fig. 1, the antenna consists of two concentric coplanar wire loops (Loop 1 and Loop 2) placed above a ground plane at a height $h$. Loop 1 (the larger one), fed at $\phi=0$ by a voltage source $V_{0}(=1$ volt $)$, acts as a primary element, while Loop 2 (the smaller one) serves as a parasitic element. In order to radiate a $\mathrm{CP}$ wave, a small gap is introduced on each loop. The size (or the radius $r_{1}$ ) of Loop 1 is determined by the lower limit of an operating frequency band (its circumference is slightly longer than one wavelength at the lower frequency). By adjusting the size (or the radius $r_{2}$ ) of Loop 2, the positions (at $\phi=\phi_{1}$ for Gap 1 and $\phi=\phi_{2}$ for Gap 2) of two gaps, and the height $h$, an optimal performance for the on axis (in the $z$ direction) axial ratio can be achieved. 


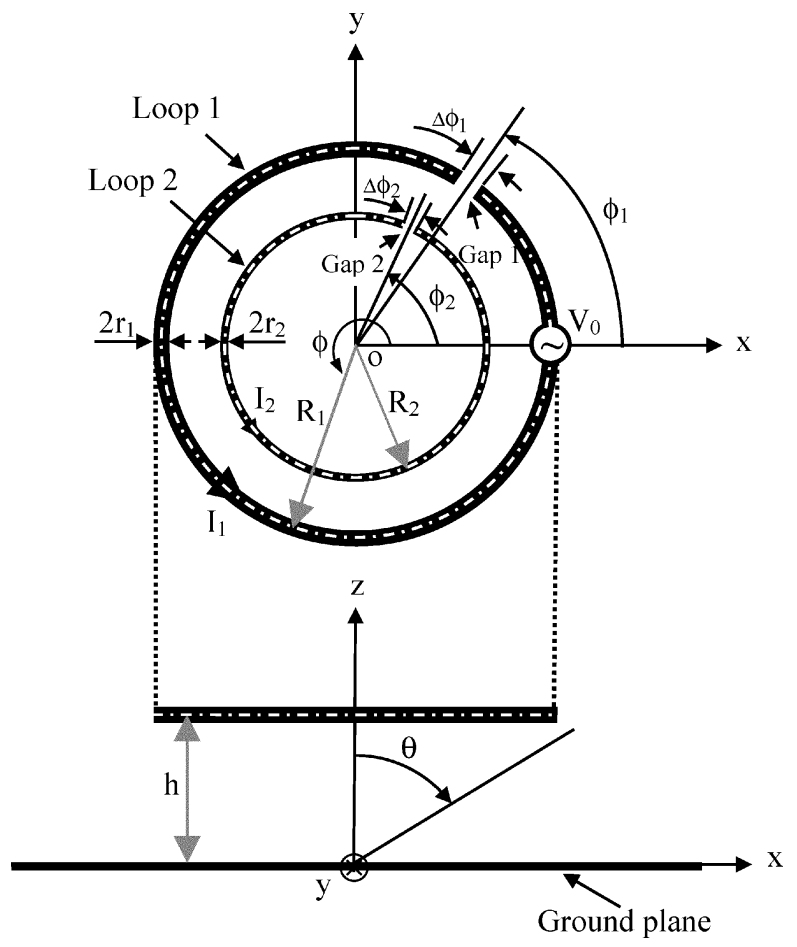

Fig. 1. Geometry of a circularly polarized circular-loop antenna with a parasitic circular loop for bandwidth enhancement.

\section{B. Numerical Results}

The circular-loop antenna with a parasitic element was numerically simulated using a Method-of-Moment based electromagnetic analysis software (NEC 1.1). In the simulation, 72 piecewise linear segments (i.e., $5^{\circ}$ per segment) were employed to model the geometry of each circular loop and an infinite ground plane was assumed. Both of the gap widths $\left(\Delta \phi_{1}\right.$ for Gap 1 and $\Delta \phi_{2}$ for Gap 2) are $5^{\circ}$ (i.e., one segment). The wire radius $\left(r_{1}\right)$ of Loop 1 is chosen to be two times the radius $\left(r_{2}\right)$ of Loop 2, which is set at $r_{2}=0.0058 \lambda_{0}$, where $\lambda_{0}$ is the free-space wavelength at the center frequency $f_{c}$. The circumference of Loop 1 is found to be $1.288 \lambda_{0}$ while the circumference of Loop 2 and the height $h$ are optimized to be $0.867 \lambda_{0}$ and $0.236 \lambda_{0}$, respectively. The optimal gap positions are found to be at $\phi_{1}=55^{\circ}, \phi_{2}=65^{\circ}$. Note that the position of Gap 1 needs to be moved to $\phi_{1}=40^{\circ}$ (for a minimum AR of $\sim 0 \mathrm{~dB}$ ) if without the parasitic element (i.e., Loop 2). As for the selection of geometric parameters of the circular loop, it will be discussed in more detail later.

Fig. 2 shows the axial ratio comparison between the circularloop antennas with and without the parasitic element. It is seen that the $\mathrm{AR}(\leq 2 \mathrm{~dB})$ bandwidth is increased from $6.5 \%$ to $20 \%$ by introducing the parasitic element. It is also observed that there are two minimum AR points appearing at a lower (than the center frequency $f_{c}$ ) frequency $f_{L}$ and at a higher frequency $f_{H}$, respectively. It is the combination of the two minimum AR points that results in the bandwidth enhancement. The AR bandwidth can be further increased at the expense of a higher maximum $\mathrm{AR}$ criterion $\left(\mathrm{AR}_{\max }\right)$.

The frequency characteristics of the input impedance and gain are presented in Fig. 3. It is seen that over the AR $\leq 2 \mathrm{~dB}$ bandwidth the gain is near $9 \mathrm{dBi}$ and the input impedance is

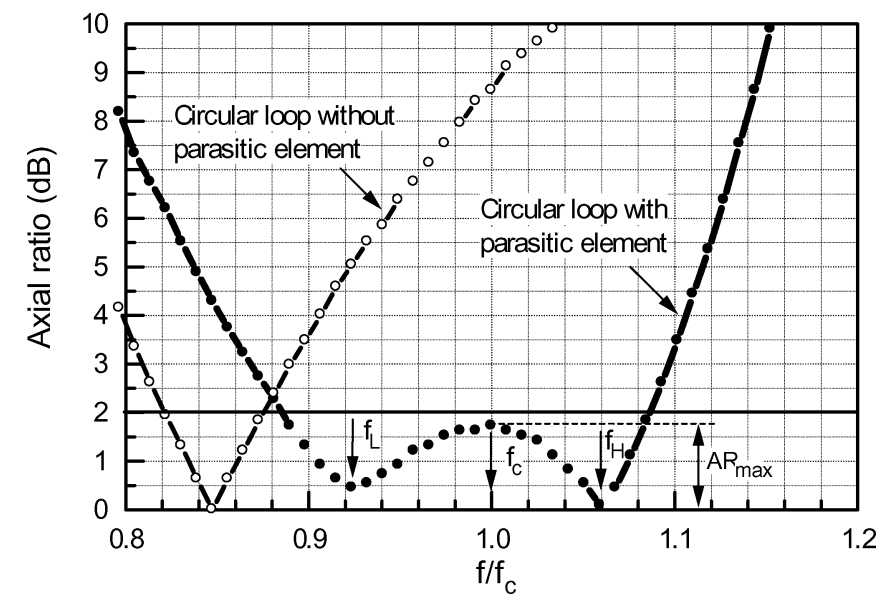

Fig. 2. Axial ratio comparison between the circular-loop antennas with and without a parasitic element. [Geometric parameters: $r_{1}=0.205 \lambda_{0}, r_{2}=$ $0.138 \lambda_{0}, h=0.236 \lambda_{0}, r_{1}=0.0118 \lambda_{0}, r_{2}=0.0059 \lambda_{0}, \phi_{1}=55^{\circ}\left(\phi_{1}=\right.$ $40^{\circ}$ if without the parasitic element), $\phi_{2}=65^{\circ}, \Delta \phi_{1}=5^{\circ}$, and $\Delta \phi_{2}=5^{\circ}$ ].

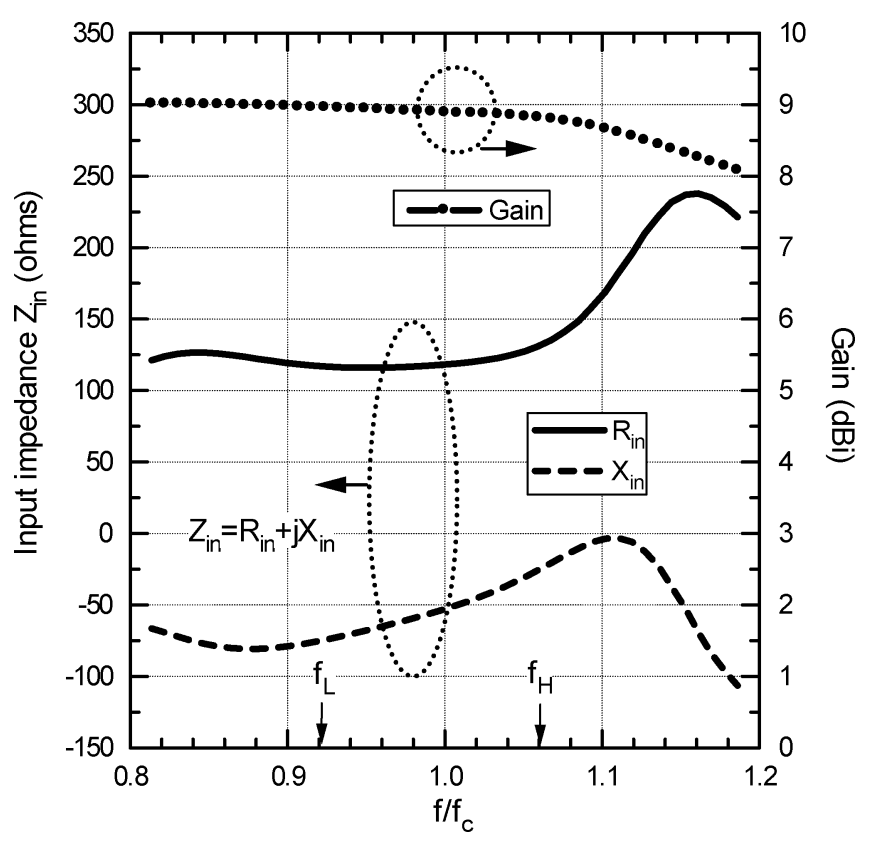

Fig. 3. Input impedance and gain of the circular-loop antenna with a parasitic element.

around $125-\mathrm{j} 50 \mathrm{ohms}$. The radiation pattern of the circular-loop antenna with the parasitic element is similar to that without a parasitic element [4].

\section{Discussion}

The current distribution on the circular loop (Loop 1) with a parasitic element (Loop 2) is plotted in Fig. 4 at $f=f_{c}$. It is observed that the current $\left(I_{1}\right)$ on Loop 1 has a considerable fluctuation in magnitude while the current $\left(I_{2}\right)$ on Loop 2 shows little feature of a traveling-wave current distribution. Fortunately, the total current $\left(I_{1}+I_{2}\right)$ has the feature of a traveling-wave current distribution with a more uniform magnitude than the current on Loop 1. This is essential for a CP radiation pattern. From this observation, we conclude that the role of the parasitic element is to compensate the magnitude variation of the current on the primary element. It should be mentioned that it is difficult 

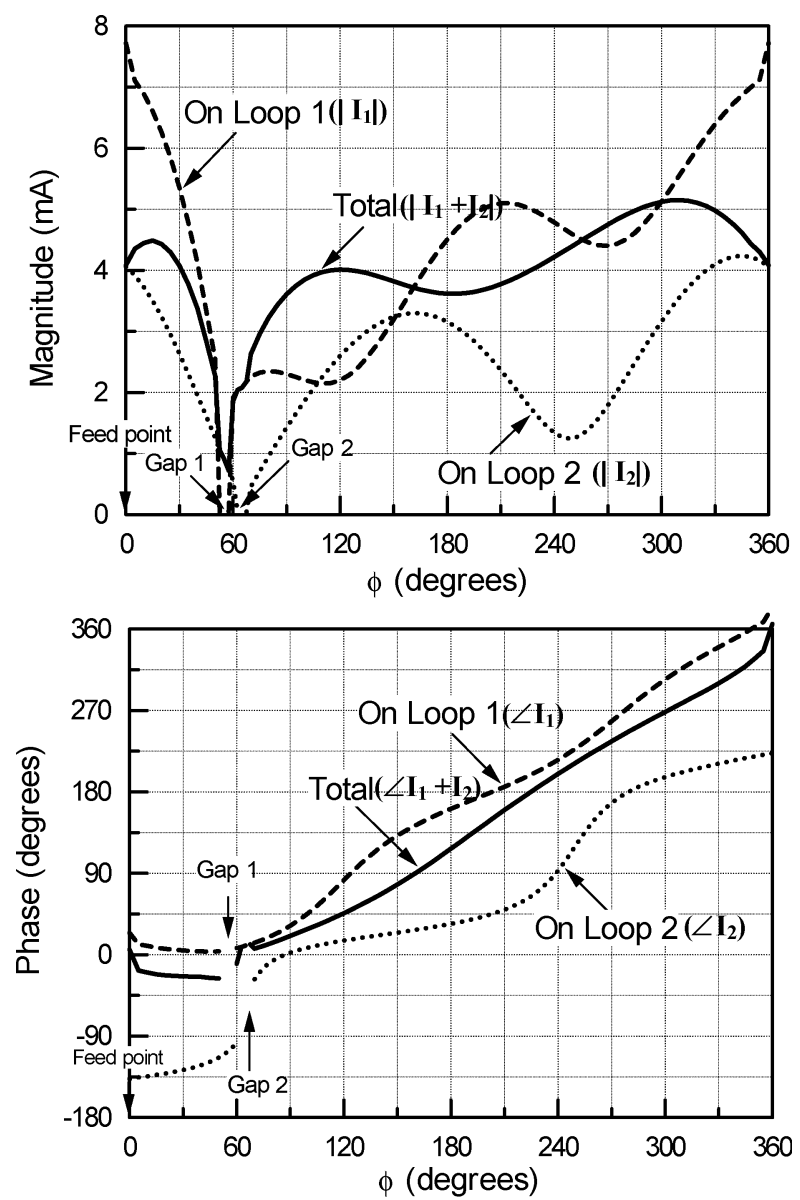

Fig. 4. Current distribution on the circular-loop antenna with a parasitic element $\left(f=f_{c}\right)$.

to enhance the AR bandwidth by placing the parasitic element outside the fed loop. The reason for this difficulty is that the current on the parasitic loop does not show a traveling-wave current distribution, making it impossible to achieve the desirable $\mathrm{CP}$ radiation.

The effect of the geometric parameters on the axial ratio of the circular-loop antenna with a parasitic element has been investigated. It is found that as the size of parasitic loop (Loop 2) increases, the minimum AR point at the higher frequency $f_{H}$ (which decreases with the increase in the size of Loop 2) moves toward the center frequency, lowering the maximum AR $\left(\mathrm{AR}_{\max }\right)$. The height $(h)$ above the ground plane affects the minimum AR points $\left(f_{L}\right.$ and $\left.f_{H}\right)$ and the $\mathrm{AR}_{\max }$ and there is an optimal value for the height at which the AR bandwidth is maximized for a certain $\mathrm{AR}_{\max }$. The positions of Gap 1 and Gap 2 mainly affect the minimum $\mathrm{AR}$ points $f_{L}$ and $f_{H}$, respectively (in turn the $\mathrm{AR}_{\max }$ ). This is because the effective length for a traveling-wave current distribution on Loop 1 [i.e., $\left(2 \pi-\phi_{1}\right) r_{1}$ ] of Loop 1 decreases as $\phi_{1}$ increases; thus $f_{L}$ increases with increasing $\phi_{1}$. On the other hand, $f_{H}$ decreases as $\phi_{2}$ decreases because the effective length on Loop2 increases. The wire radii (i.e., $r_{1}$ and $\left.r_{2}\right)$ of the loops and the widths $\left(\Delta \phi_{1}\right.$ and $\left.\Delta \phi_{2}\right)$ of the gaps also have some effects on the AR performance but are not very critical. [Note that in this paper we only consider the thin-wire case, i.e., $2 \pi r_{1}$ (and $r_{2}$ ) $<0.1 \lambda_{0}$.] As a rule of thumb, it is suggested to choose $r_{1}=0.012-0.008 \lambda_{0}, r_{2}=0.3-0.5 r_{1}$, and the gap widths of 2-4 times the wire radii.

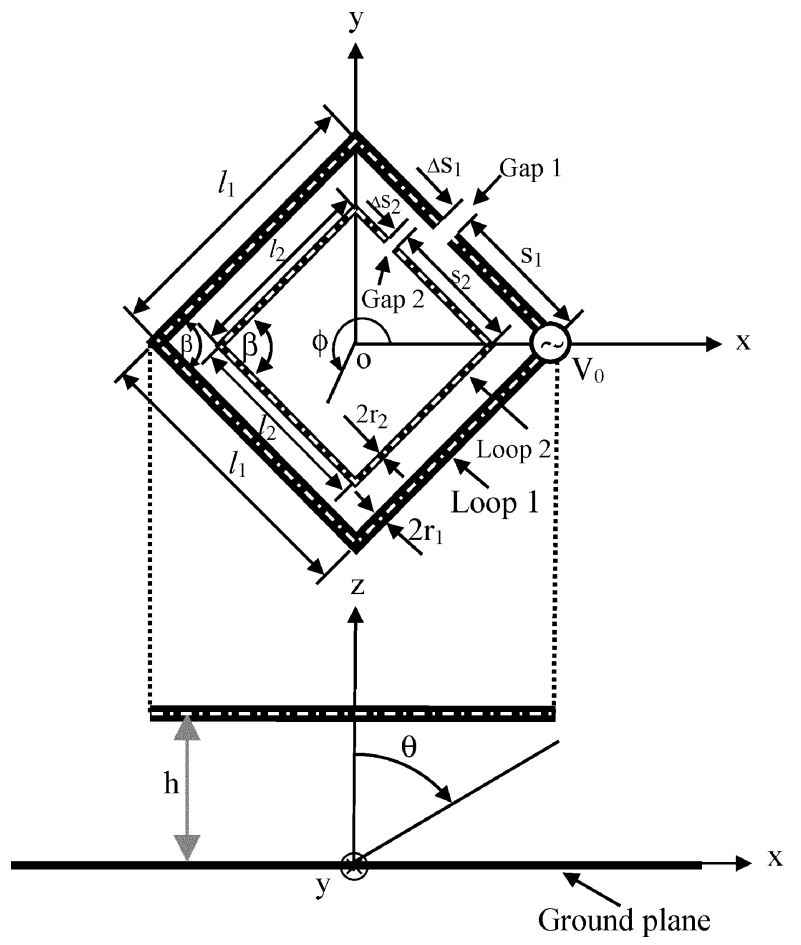

Fig. 5. Geometry of a circularly polarized rhombic-loop antenna with a parasitic rhombic loop.

\section{RhOMBIC-LOOP ANTENNA With A PARASITIC ELEMENT}

It has been demonstrated in [2] that it is possible for a rhombic-loop antenna to achieve an AR bandwidth of more than $20 \%$ ( $\mathrm{AR} \leq 2 \mathrm{~dB})$ by adjusting the rhombus vertex angle. In this section, it will be shown that the AR bandwidth can be increased to $40 \%$ by adding a parasitic element. Fig. 5 illustrates the geometry of the rhombic-loop antenna with a parasitic similar rhombic loop where the rhombus vertex angle is denoted by $\beta$. For a certain $\beta$, an optimal AR performance is obtained by slightly adjusting the height $h$. The procedure for determining other geometric parameters of the rhombic loop is similar to that of the circular loop. The perimeters of the primary and parasitic loops (i.e., Loop 1 and Loop 2 indicated in Fig. 5) are found to be $1.396 \lambda_{0}$ and $0.872 \lambda_{0}$, respectively, and are fixed at the same values as $\beta$ varies. The gap positions are adjusted to be $s_{1}=0.186 \lambda_{0}$ for Gap 1 and $s_{2}=0.174 \lambda_{0}$ for Gap 2.

Fig. 6 shows the AR of the rhombic-loop antenna at different rhombus vertex angles ranging from $\beta=90^{\circ}$ to $\beta=45^{\circ}$. It is clearly seen that the AR bandwidth increases as $\beta$ decreases. The AR $(\leq 2 \mathrm{~dB})$ bandwidth is about $24 \%$ at $\beta=90^{\circ}$ and is increased to more than $40 \%$ at $\beta=45^{\circ}$. The bandwidth variation with the rhombus vertex angle is an inherent property of the rhombic-loop antennas, which has also been observed for the impedance and radiation characteristics [1], [2].

Fig. 7 displays the gain of the rhombic-loop antenna as a function of frequency at different rhombus vertex angles. It is indicated that the gain decreases with the increase of the frequency and the decrease of the rhombus vertex angle. Therefore, there is a tradeoff between the bandwidth for circular polarization and the gain. The loss of gain comes from two aspects: 1) the shrinkage of antenna aperture (i.e., the cross section area of 


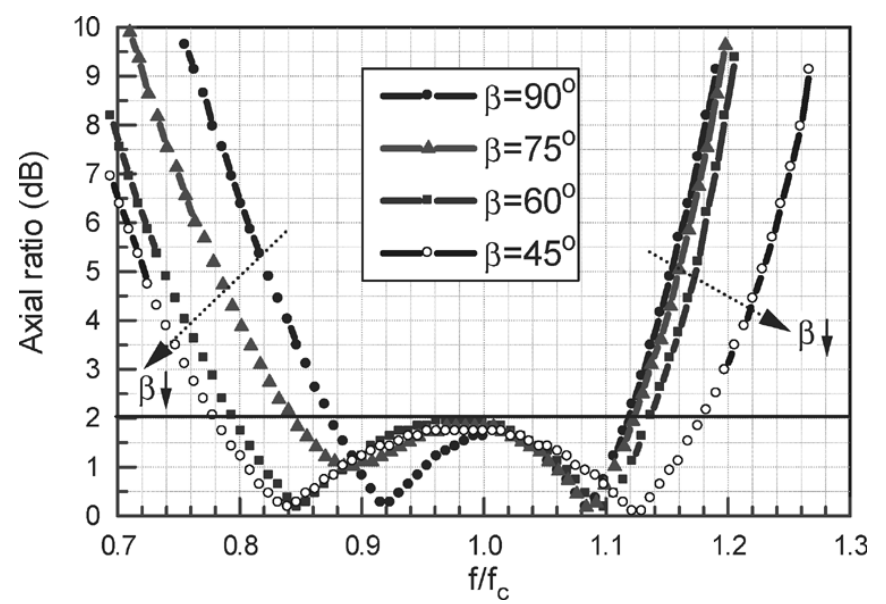

Fig. 6. Axial ratio of the rhombic-loop antenna with a parasitic element at different rhombus vertex angles. (Geometric parameters: $l_{1}=0.349 \lambda_{0}, l_{2}=$ $0.218 \lambda_{0}, s_{1}=0.186 \lambda_{0}, s_{2}=0.174 \lambda_{0}, \Delta s_{1}=0.0232 \lambda_{0}, \Delta s_{2}=0.0145 \lambda_{0}$, $r_{1}=0.0118 \lambda_{0}, r_{2}=0.0059 \lambda_{0}, h=0.262 \lambda_{0}$ for $\beta=90^{\circ}, h=0.284 \lambda_{0}$ for $\beta=75^{\circ}, h=0.306 \lambda_{0}$ for $\beta=60^{\circ}$, and $h=0.328 \lambda_{0}$ for $\beta=45^{\circ}$ ).

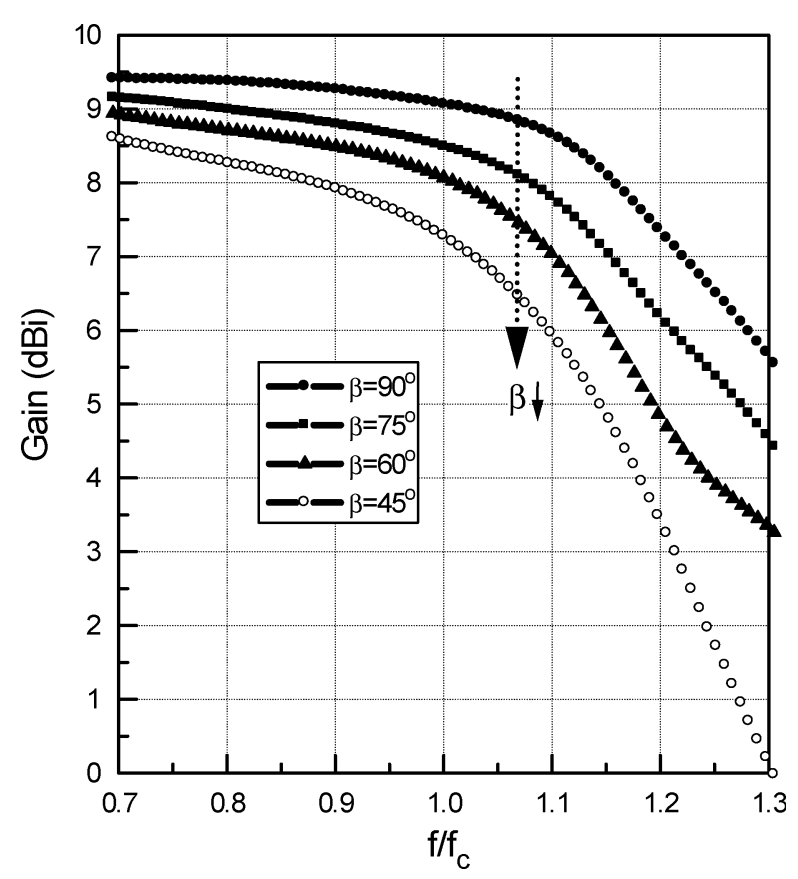

Fig. 7. Gain of the rhombic-loop antenna with a parasitic element at different rhombus vertex angles.

the rhombic loop) as $\beta$ decreases and 2) the increase in the electrical height of the rhombic loops above the ground plane. Note that the directivity of a horizontal electric dipole decreases as its height (increased from $0.262 \lambda_{0}$ to $0.328 \lambda_{0}$ as $\beta$ decreases from $90^{\circ}$ to $45^{\circ}$ ) above the ground plane increases [1]. The radiation pattern of the rhombic-loop antenna with a parasitic element is very similar to that of a circular-loop antenna.

\section{DUAL RHOMBIC-LOOP ANTENNA With A PARASITIC ELEMENT}

The dual rhombic-loop antenna that consists of two rhombic loops was developed in [3] with two types of feed configurations: i) series feed and ii) parallel feed. An AR bandwidth of
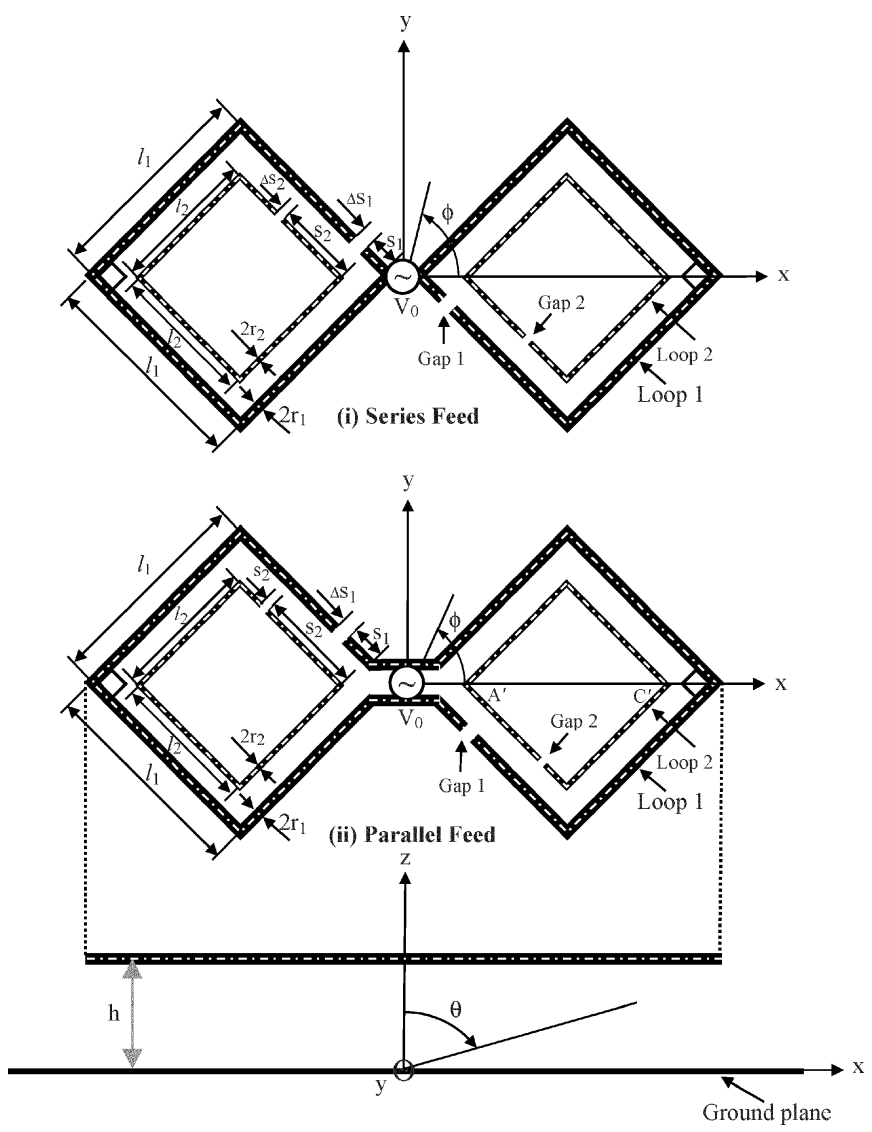

Fig. 8. Geometry of series- and parallel-fed dual rhombic-loop antennas with a parasitic dual rhombic loop.

more than $20 \%(\mathrm{AR} \leq 2 \mathrm{~dB}$ ) has been obtained for a parallel-fed dual rhombic-loop antenna [3]. In this section, it will be demonstrated that an AR bandwidth of near $40 \%$ for $\mathrm{AR} \leq$ $1 \mathrm{~dB}$ can be achieved for the parallel-fed dual rhombic-loop antenna by adding a parasitic element (also a dual rhombic loop). Fig. 8 depicts the geometry of a dual rhombic-loop antenna with i) a series feed and ii) a parallel feed. The rhombus vertex angle is fixed at $\beta=90^{\circ}$ because it has a higher gain and a less dramatic impedance variation than the rhombic loop with a smaller vertex angle (as shown in the previous section). A similar rhombic loop (Loop 2) is placed inside of each of the two primary rhombic loops (Loop 1). There is a small gap (< $0.025 \lambda_{0}$ ) along each of the loops. Like the single-loop antennas described previously, an optimal AR performance of the dual rhombic-loop antenna can be obtained by adjusting the size of the parasitic element, the positions of the gaps, and the height of the loops above the ground plane. By simulation, it is found that the best position of Gap 1 for the series feed is close to the feed point, i.e., $s_{1}=0$. Therefore, if without the parasitic element the series-fed dual rhombic-loop antenna is essentially an S-type [13] or a figure-of-eight wire antenna [14]. The geometric parameters of the dual rhombic-loop antenna are obtained by numerous simulations. We simulated the antenna usually following this procedure: first, the loop antenna without a parasitic element was simulate and the first minimum $\operatorname{AR}(<0.5$ $\mathrm{dB}$ ) point near the lower limit of the design frequency band was found by adjusting the loop size and the gap position; next, a similar parasitic element was added inside the original loop and 


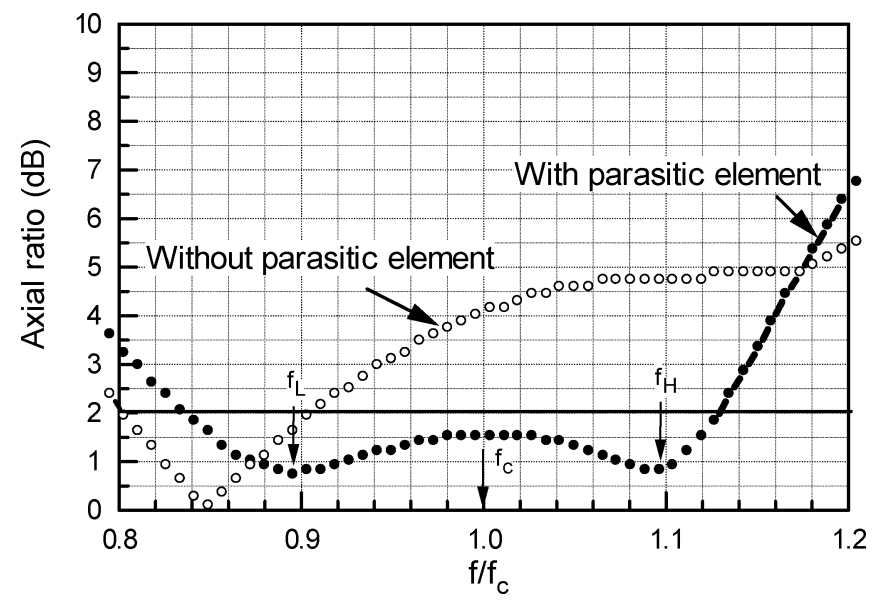

Fig. 9. Axial ratio comparison between the series-fed dual rhombic-loop antennas with and without a parasitic element. (Geometric parameters: $l_{1}=0.345 \lambda_{0}, l_{2}=0.216 \lambda_{0}, s_{1}=0, s_{2}=0.158 \lambda_{0}, \Delta s_{1}=0.023 \lambda_{0}$, $\Delta s_{2}=0.0105 \lambda_{0}, r_{1}=0.0086 \lambda_{0}, r_{2}=0.0043 \lambda_{0}, h=0.28 \lambda_{0}$, and $\beta=90^{\circ}$.

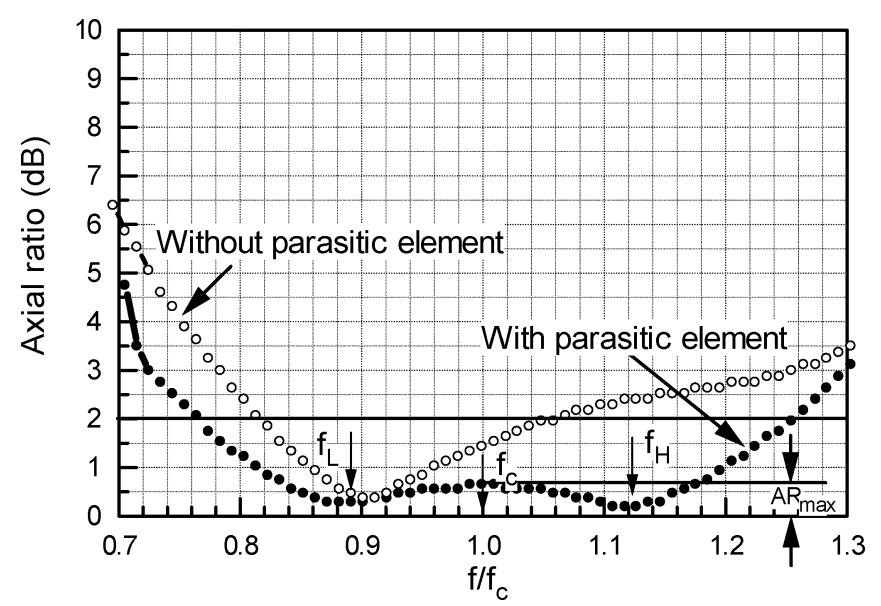

Fig. 10. Axial ratio comparison between the parallel-fed dual rhombic-loop antennas with and without a parasitic element. [Geometric parameters: $l_{1}=$ $0.272 \lambda_{0}, l_{2}=0.17 \lambda_{0}, s_{1}=0.072 \lambda_{0}\left(s_{1}=0.018 \lambda_{0}\right.$ if without the parasitic element), $s_{2}=0.125 \lambda_{0}, \Delta s_{1}=0.018 \lambda_{0}, \Delta s_{2}=0.011 \lambda_{0}, r_{1}=0.01 \lambda_{0}$, $r_{2}=0.005 \lambda_{0}, h=0.28 \lambda_{0}$, and $\beta=90^{\circ} \mathrm{]}$.

the size of the parasitic element was kept changing until the second minimum AR point appears around the upper limit of the design frequency band; finally, the values of AR at two minimum AR points and at the center frequency were tuned below the maximum AR criterion (e.g., $2 \mathrm{~dB}$ ) by slightly adjusting the gap positions, the sizes of two loops, the height above the ground plane. It is found that the outer perimeters $\left(8 l_{1}\right)$ of the series-fed and parallel-fed dual rhombic-loop antennas are $\sim 2.8 \lambda_{0}$ and $\sim 2.2 \lambda_{0}$, respectively.

Fig. 9 shows the AR comparison between the series-fed dual rhombic-loop antennas with and without a parasitic element. We can see that the bandwidth for $\mathrm{AR} \leq 2 \mathrm{~dB}$ is increased from $12 \%$ to $30 \%$ by adding the parasitic element. For the parallel feed, the AR is compared in Fig. 10 between the dual rhombic-loop antennas with and without the parasitic element. It is observed that the $\mathrm{AR} \leq 2 \mathrm{~dB}$ bandwidth is increased from $25 \%$ to $50 \%$ by introducing the parasitic element. More importantly, a very low maximum $\mathrm{AR}\left(\mathrm{AR}_{\max }<0.75 \mathrm{~dB}\right)$ is obtained, and it is seen that the AR bandwidth for $\mathrm{AR} \leq 1 \mathrm{~dB}$

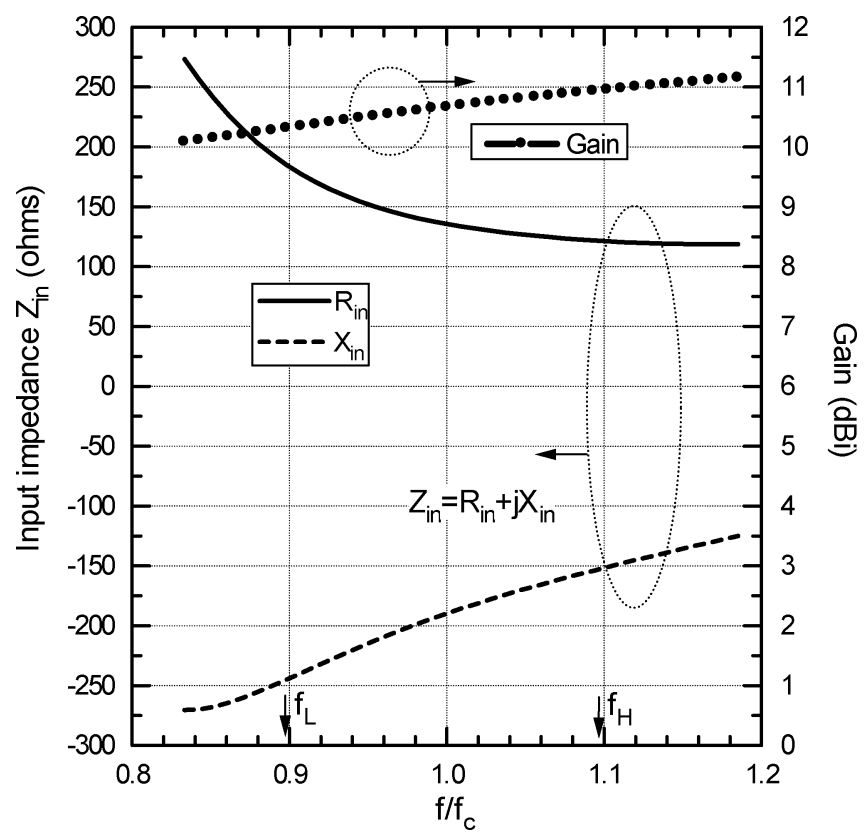

Fig. 11. Input impedance and gain of the series-fed dual rhombic-loop antenna with a parasitic element.

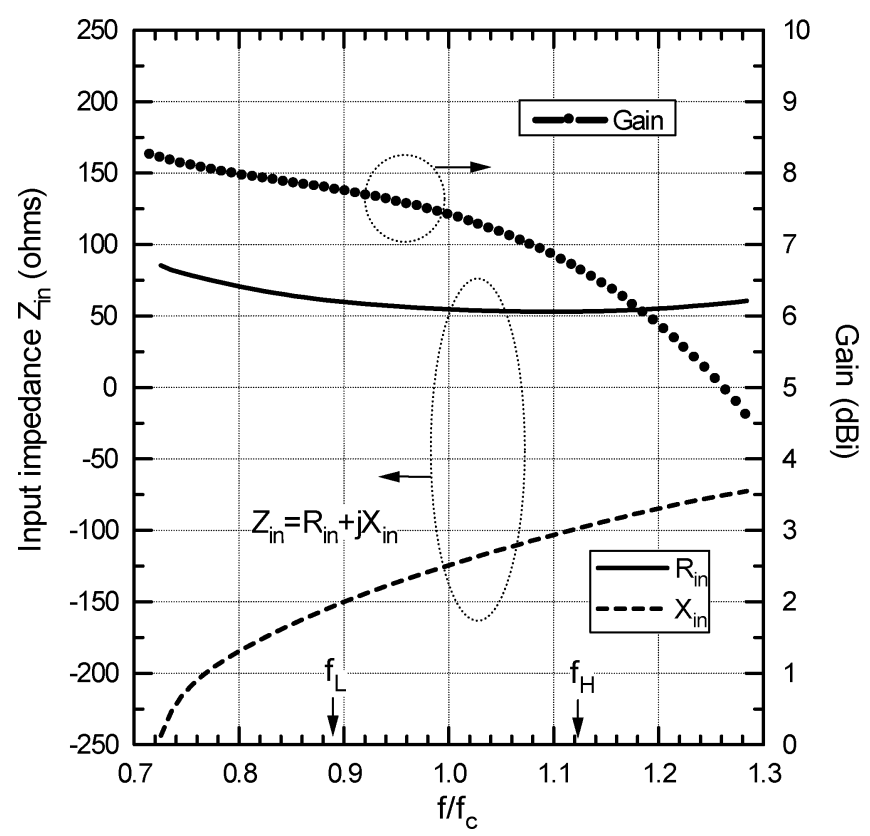

Fig. 12. Input impedance and gain of the parallel-fed dual rhombic-loop antenna with a parasitic element.

is near $40 \%$. The reason why the parallel feed corresponds to a wider bandwidth than does the series feed is that the parallel-fed dual rhombic loop is adjusted a larger height above the ground plane, which in turn increases the volume occupied by the antenna.

Figs. 11 and 12 show the frequency characteristics of the input impedance and gain of the series- and parallel-fed dual rhombic loop antennas with a parasitic element. As expected, the parallel feed has a lower input resistance (about $50 \mathrm{ohms}$ ) than that of the series feed ( $<100 \mathrm{ohms})$. It is also observed that the series feed corresponds to a higher gain (about 10-11 dBi) than does the parallel feed (varying from $8 \mathrm{dBi}$ to $6 \mathrm{dBi}$ in the $\mathrm{AR} \leq 1 \mathrm{~dB}$ bandwidth). Therefore, the series feed is suitable 
for the applications in which the gain is most important while the parallel feed can find applications where the purity of polarization is the first priority. It should be mentioned that the series feed has another important feature: the series feed can be realized by a coplanar strip line, making it possible to integrate an antenna array with feeding structure in a coplanar circuit [7], [8]. The radiation patterns of the series- and parallel-fed dual rhombic loop antennas have no significant change after the addition of a parasitic element. Usually the parallel feed corresponds to a broader beamwidth than does the series feed; hence the parallel feed has a lower gain. The broader beamwidth for the parallel feed is due to two factors: 1$)$ the smaller loop size ( $l_{1}=0.272 \lambda_{0}$ for the parallel feed versus $l_{1}=0.345 \lambda_{0}$ for the series feed) and 2) the larger height ( $h=0.323 \lambda_{0}$ for the parallel feed versus $h=0.28 \lambda_{0}$ for the series feed).

\section{EXPERIMENTAL RESULTS}

To confirm the bandwidth enhancement by the parasitic element, a broad-band circular-loop antenna and a broad-band series-fed dual rhombic-loop antenna were designed, fabricated, and measured. The reason for choosing the two configurations as examples is that the bandwidths of these antennas are within the frequency range our antenna measurement system can cover. The antenna measurement system employed is the NSI nearfield system which is capable of measuring amplitude and phase patterns. The probe used (as a TX source antenna) is a WR159 open-ended waveguide probe that covers the frequency range 4.9-7.05 GHz. Both antennas were designed on a thin dielectric substrate (substrate thickness $=0.254 \mathrm{~mm}$ ) with a low dielectric constant (RT/duroid 5880, $\left.\varepsilon_{r}=2.2\right)$ and were simulated by Microstripes 6.5-a TLM (Transmission-Line Matrix) based full-wave EM (electromagnetic) design tool. The circular-loop antenna was fed by a quarter-wavelength folded balun while the series-fed dual rhombic-loop antenna was fed by a broad-band balun for a good input impedance matching.

\section{A. Broad-Band Circular-Loop Antenna}

The geometry of the printed broad-band CP circular-loop antenna is depicted in Fig. 13. The central design frequency of this antenna is $5.75 \mathrm{GHz}$. The dimensions of the primary loop (i.e., Loop 1) and the parasitic loop (i.e., Loop 2) are indicated in the figure. The gaps on Loops 1 and 2 are located $45^{\circ}$ and $60^{\circ}$ away from the feed point, respectively. The printed circular-loop antenna is mounted $13 \mathrm{~mm}$ above a $40 \mathrm{~mm} \times 40 \mathrm{~mm}$ aluminum plate (as a finite ground plane) with the support of a piece of Styrofoam $\left(\varepsilon_{r} \cong 1.03\right)$. A quarter-wavelength folded balun [15] was used to provide a balanced feed for the antenna. Considering the narrow bandwidth of the quarter-wave balun, we employed two folded baluns: one has a length of $14 \mathrm{~mm}$ used for the frequency range 5.0-5.75 GHz and the other has a length of $12 \mathrm{~mm}$ for the band of 5.75-6.5 GHz.

Fig. 14 shows the simulated and measured results for the on axis axial ratio and gain of the broad-band circular-loop antenna. Even though the measured axial ratio is higher than the simulated result, the broad-band performance is still observed: the

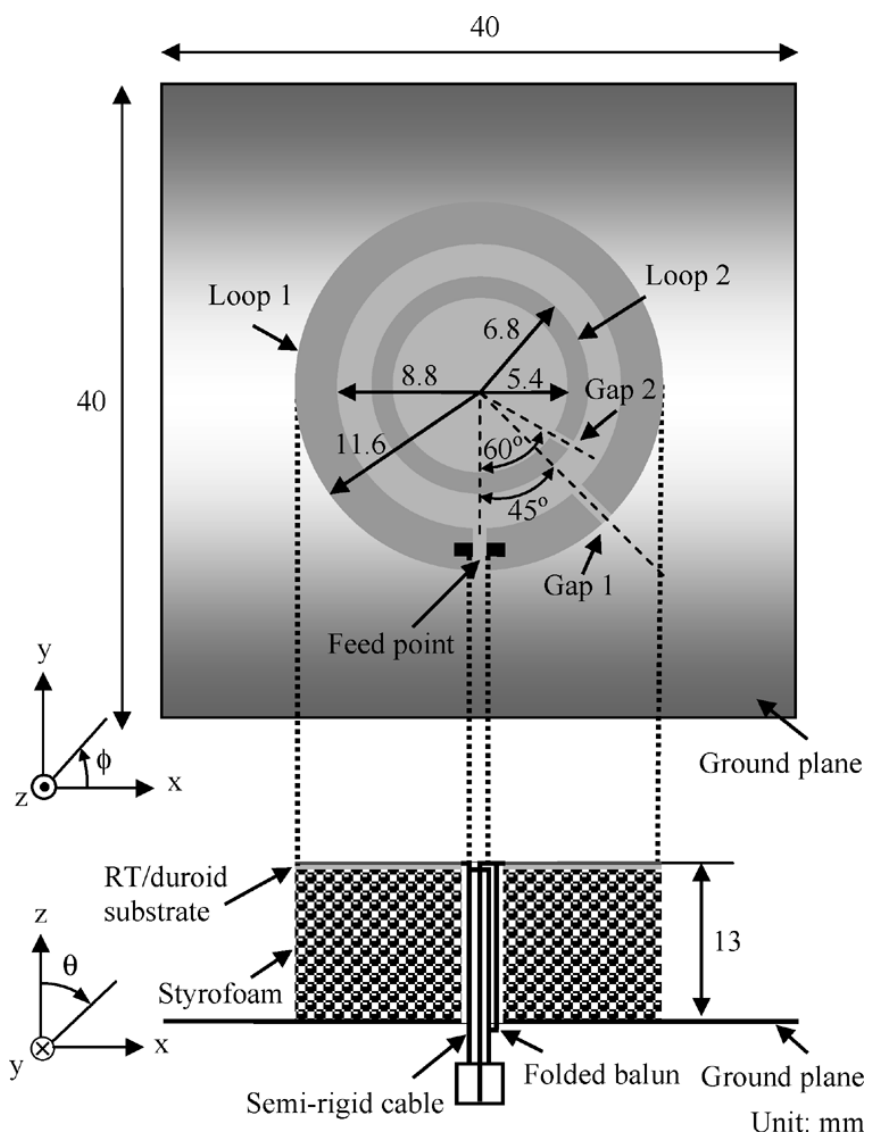

Fig. 13. Printed broad-band circularly polarized circular-loop antenna. (The width of Gap 1 is $1.0 \mathrm{~mm}$ and the width of Gap 2 is $0.6 \mathrm{~mm}$ ).

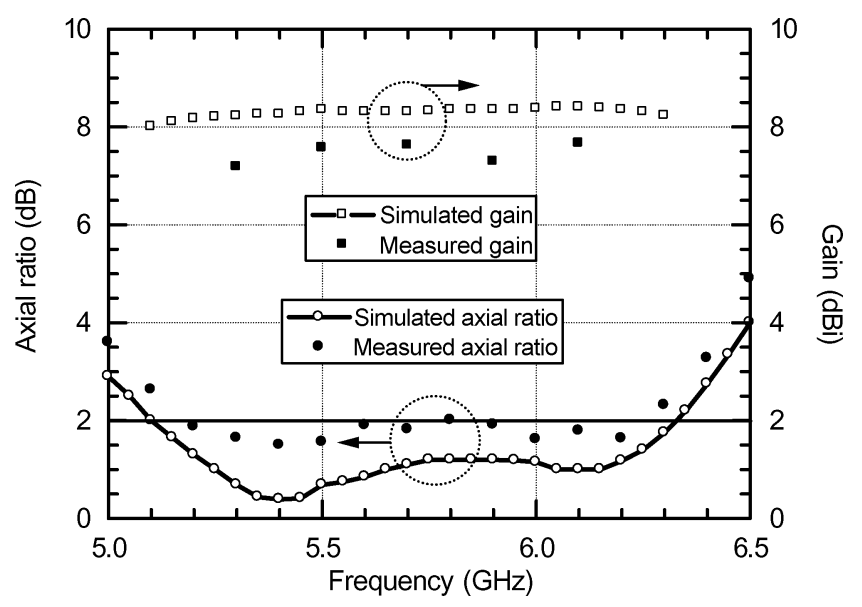

Fig. 14. Axial ratio and gain of the printed broad-band circularly polarized circular-loop antenna.

measured and simulated bandwidths for $\mathrm{AR} \leq 2 \mathrm{~dB}$ are $\sim 18 \%$ and $\sim 21 \%$, respectively. The difference between the simulated and measured results is caused by the feeding structure, which was not taken into account in the simulation. The gain of the antenna is $7-8 \mathrm{dBi}$, slightly lower than that shown in Fig. 3 due to the effect of the finite ground plane. This antenna has a voltage standing-wave ratio (VSWR) of around 3; thus an impedance matching circuit may be needed for some practical applications. 


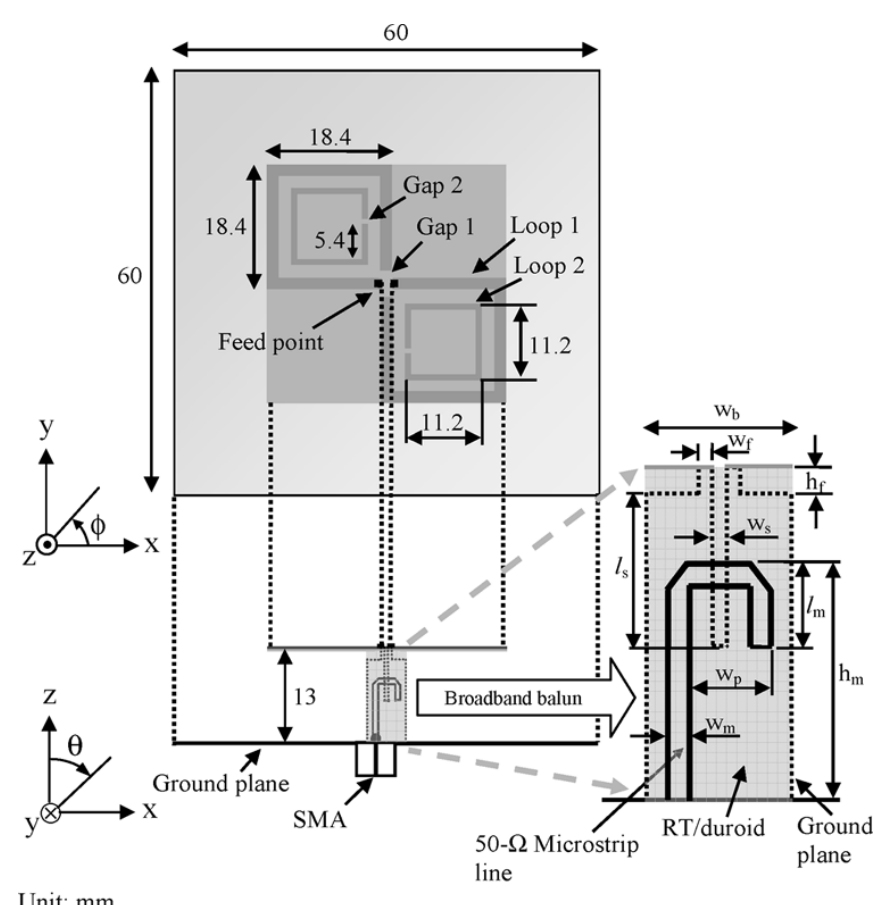

Unit: $\mathrm{mm}$

Fig. 15. Printed broad-band series-fed dual rhombic-loop antenna with a broad-band balun. (The strip width of Loop 1 is $1.6 \mathrm{~mm}$, the strip width of Loop 2 is $0.8 \mathrm{~mm}$, the width of Gap 1 is $1.0 \mathrm{~mm}$, and the width of Gap 2 is $0.6 \mathrm{~mm}$; $w_{b}=6.0 \mathrm{~mm}, w_{f}=0.6 \mathrm{~mm}, w_{s}=0.5 \mathrm{~mm}, w_{m}=0.8 \mathrm{~mm}, w_{\mathrm{p}}=3.2 \mathrm{~mm}$, $\left.h_{f}=1.0 \mathrm{~mm}, h_{m}=9.2 \mathrm{~mm}, l_{s}=6.0 \mathrm{~mm}, l_{m}=3.2 \mathrm{~mm}\right)$.

\section{B. Broad-Band Series-Fed Dual Rhombic-Loop Antenna}

The configuration of the printed broad-band CP dual rhombic-loop antenna is illustrated in Fig. 15. The central design frequency of this antenna is set at $6.0 \mathrm{GHz}$. The printed dual rhombic-loop antenna with parasitic element is also mounted $13 \mathrm{~mm}$ above a $60 \mathrm{~mm} \times 60 \mathrm{~mm}$ aluminum plate with the support of a broad-band balun which was fabricated on the same type of RT/duroid substrate as used for the printed loop (namely, $\varepsilon_{r}=2.2$ and thickness $=0.254 \mathrm{~mm}$ ). This type of broad-band balun has been widely used for broad-band printed dipole antennas [16]-[18]. To our knowledge, however, this is the first time to be used for a CP loop antenna. The broad-band balun can excite the balanced mode by making use of the coupling between the microstrip line printed on one side of the substrate to the slot which is etched on the other side (which also serves as the ground plane for the microstrip line) of the substrate. A good impedance matching can be achieved by adjusting the length $\left(l_{s}\right)$ of the slot, the height $\left(h_{m}\right)$ and the length $\left(l_{m}\right)$ of the microstrip line. Fig. 16 presents the simulated and measured results for VSWR of the broad-band balun fed dual rhombic-loop antenna, which shows a broad-band impedance bandwidth (VSWR $<1.8$ ) of $~ 38 \%$ from 5.0-7.4 GHz. (Note that the broad-band balun was included in the simulation.)

The simulated and measured results for the axial ratio and gain are compared in Fig. 17, showing good agreement. The AR bandwidth for $\mathrm{AR} \leq 2 \mathrm{~dB}$ is found to be about $30 \%$, which implies that the broad-band balun has no significant effect on the broad-band performance for circular polarization of the dual rhombic-loop antenna. The measured gain is near 10 $\mathrm{dBi}$. The radiation patterns simulated and measured in two principal planes $\left(\phi=45^{\circ}\right.$ and $\left.\phi=135^{\circ}\right)$ are plotted in Fig. 18 at

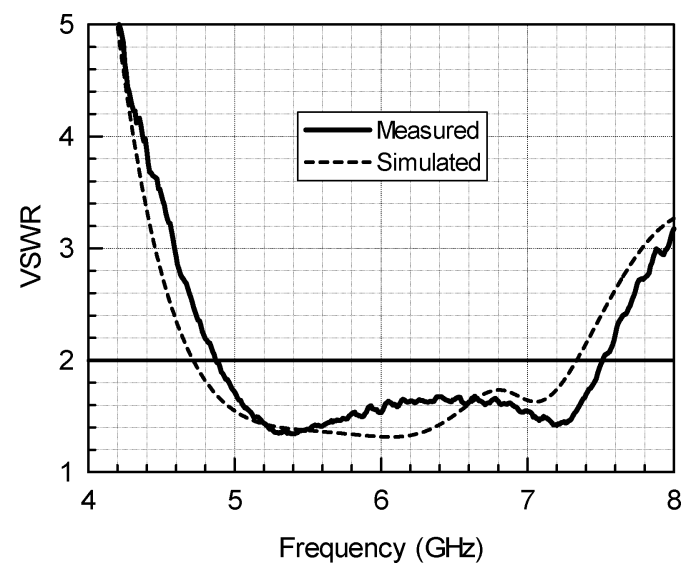

Fig. 16. VSWR of the printed broad-band series-fed dual rhombic-loop antenna with a broad-band balun.

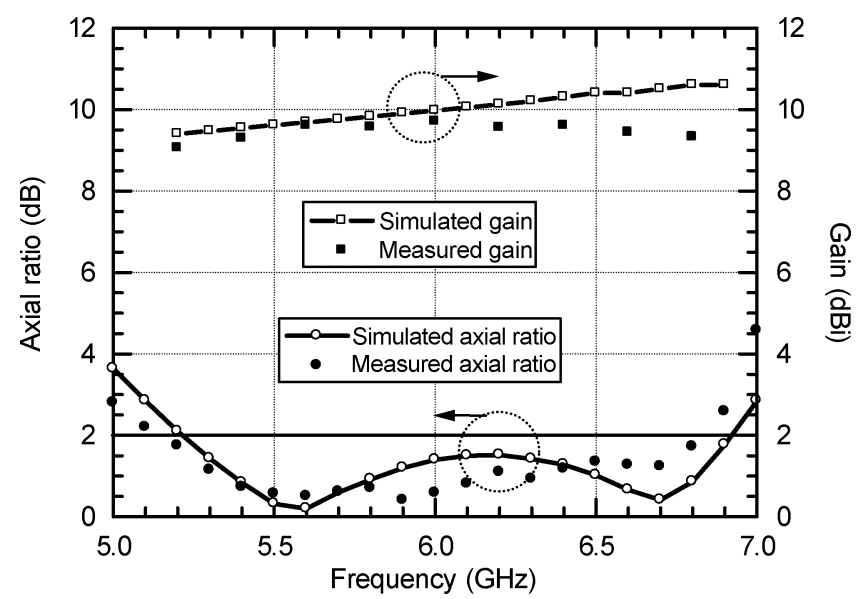

Fig. 17. Axial ratio and gain of the printed broad-band series-fed dual rhombic-loop antenna.

5.4, 6.0, and 6.6 GHz. Good agreement is observed in the main beam of the co-polarization (i.e., the left-hand circular polarization, LHCP). The level of cross-polarization (i.e., the right-hand circular polarization, RHCP) is lower than $-15 \mathrm{~dB}$ over the main beam. It is also noticed that the beamwidth in the plane of $\phi=45^{\circ}$ is much broader than that in plane of $\phi=135^{\circ}$. This is because the antenna profile along the direction of $\phi=135^{\circ}$ is much longer than that along the direction of $\phi=45^{\circ}$.

It should be mentioned that we tried to use this type of broadband balun for the feeding of the circular-loop antenna, but it failed. Our investigation shows that this type of broad-band balun is not suitable for an asymmetric (with respect to the feed point) $\mathrm{CP}$ antenna structure (such as the circular loop shown in Fig. 1 or the rhombic loop in Fig. 5). It is possible to achieve a broad-band impedance matching, but the CP performance can be significantly degraded. The reason is that the asymmetric antenna structure may cause an unbalanced current on the feeding structure, thus contributing to the radiation pattern, introducing an unwanted cross-polarized field component.

\section{CONCLUSION}

It has been demonstrated that the bandwidth for circular polarization of a loop antenna can be significantly increased by 

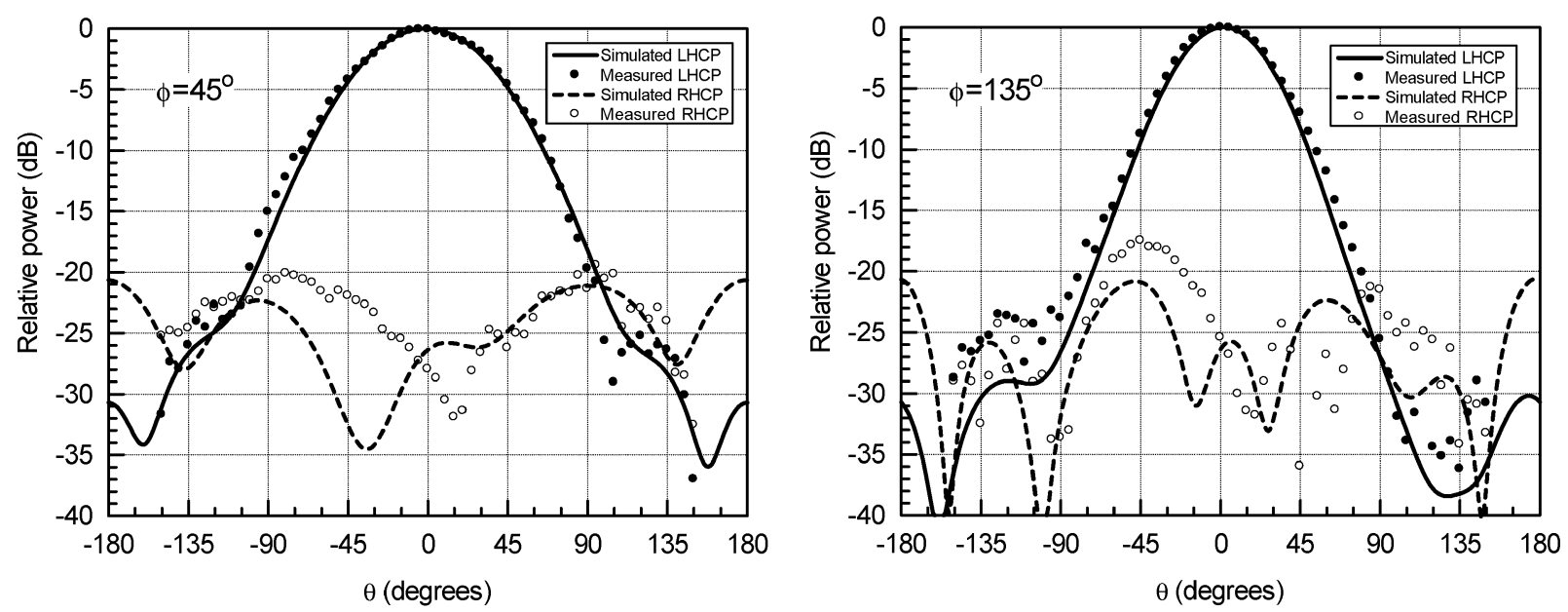

(a)
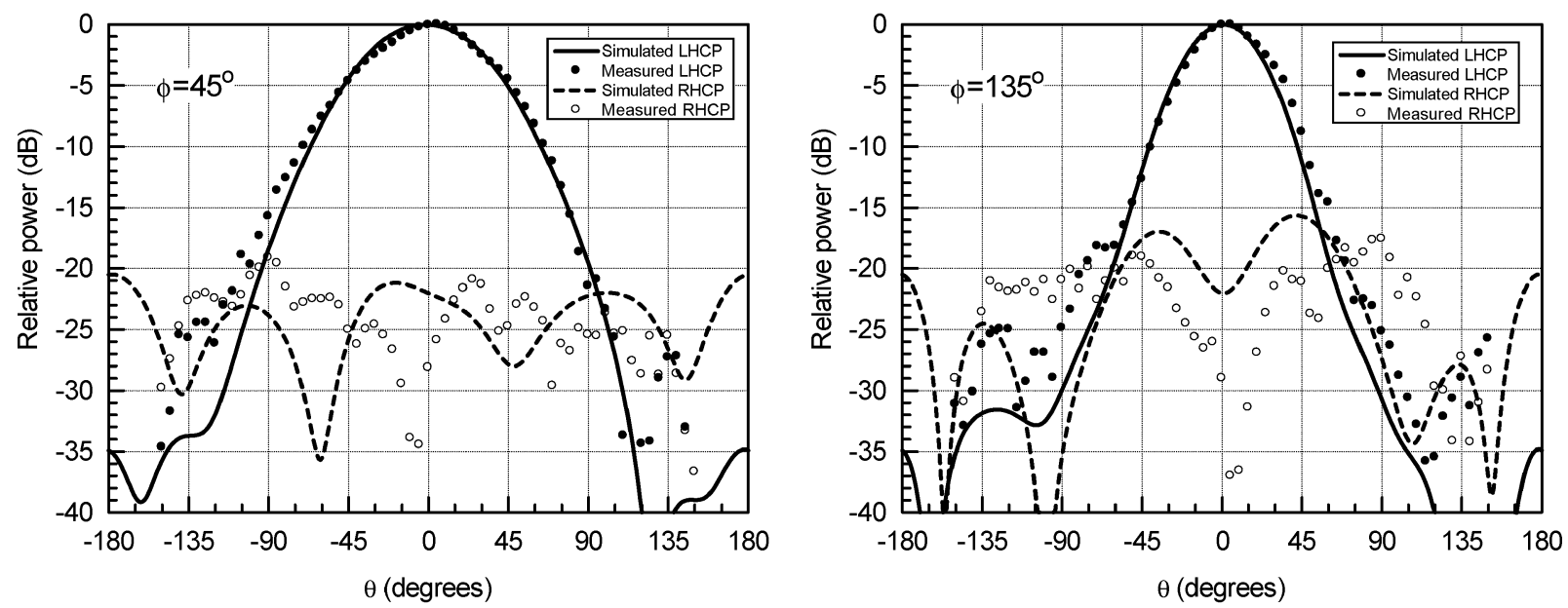

(b)
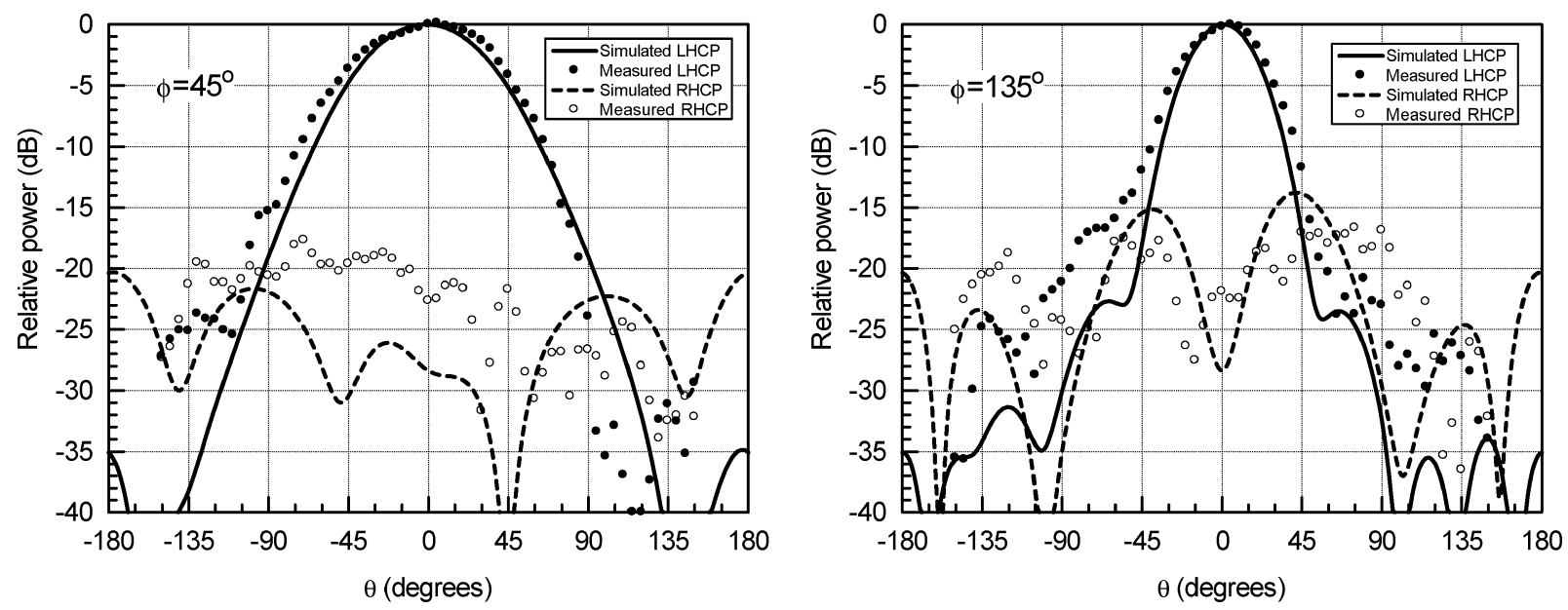

(c)

Fig. 18. Radiation patterns of the printed broad-band series-fed dual rhombic-loop antenna. (a) $f=5.4 \mathrm{GHz}$. (b) $f=6.0 \mathrm{GHz}$. (c) $f=6.6 \mathrm{GHz}$.

incorporating a similar loop inside the original loop. The incorporated loop serves as a parasitic element and introduces an additional minimum axial ratio (AR) point. A combination of the original and additional minimum AR points leads to an AR bandwidth enhancement. It has been found that the AR ( $\leq 2 \mathrm{~dB})$ bandwidth of the circular-loop antenna with a parasitic circular loop can be increased from $6.5 \%$ (without the par- asitic element) to $20 \%$. A rhombic-loop antenna with a parasitic rhombic loop can achieve an AR bandwidth $(\mathrm{AR} \leq 2 \mathrm{~dB})$ of more than $40 \%$ at a rhombus vertex angle of $45^{\circ}$. A bandwidth of $30 \%(\mathrm{AR} \leq 2 \mathrm{~dB})$ with a gain of $10 \mathrm{dBi}$ has been obtained for a series-fed dual rhombic-loop antenna with a parasitic dual rhombic loop while a bandwidth of $50 \%$ for $\mathrm{AR} \leq 2 \mathrm{~dB} \mathrm{~dB}$ and a bandwidth of $40 \%$ for $\mathrm{AR} \leq 1 \mathrm{~dB}$ have been realized 
for the parallel-fed dual rhombic loop by adding a parasitic element. The experimental results from a circular-loop antenna and a series-fed dual rhombic-loop antenna have confirmed the bandwidth enhancement. A good impedance matching has been achieved for the series-fed dual rhombic-loop antenna by introducing a broad-band balun. The broad-band circularly polarized loop antennas have two important features: i) it is easy to change the sense of circular polarization (from left-hand to right-hand, and vice versa) using radio-frequency (RF) switches such as microelectro-mechanical systems (MEMS), optoelectronic switches, or PIN diodes; ii) it is able to implement an antenna array in a coplanar stripline circuit.

\section{ACKNOWLEDGMENT}

The authors wish to acknowledge the support of the Georgia Electronic Design Center (GEDC) and the NSF Packaging Research Center. They would also like to thank the Reviewers for their valuable suggestions.

\section{REFERENCES}

[1] C. A. Balanis, Antenna Theory: Analysis and Design. New York: Wiley, 1997, pp. 203-248.

[2] H. Morishita and K. Hirasawa, "Wideband circularly-polarized loop antenna," in Proc. IEEE AP-S Int. Symp., 1994, pp. 1286-1289.

[3] H. Morishita, K. Hirasawa, and T. Nagao, "Circularly polarized wire antenna with a dual rhombic loop," Proc. Inst. Elect. Eng. Microw. Antennas Propag., pt. H, vol. 145, no. 3, pp. 219-224, Jun. 1998.

[4] R. L. Li, V. Fusco, and H. Nakano, "Circularly polarized open-loop antenna," IEEE Trans. Antennas Propag., vol. 51, no. 9, pp. 2475-2477, Sep. 2003.

[5] M. Sumi, K. Hirasawa, and S. Shi, "Two rectangular loops fed in series for broad-band circular polarization and impedance matching," IEEE Trans. Antennas Propag., vol. 52, no. 2, pp. 551-554, Feb. 2004.

[6] W. L. Curtis, "Spiral antennas," IRE Trans. Antennas Propag., vol. 8, pp. 298-306, May 1960.

[7] B. Strassner and K. Chang, "5.8-GHz circularly polarized dual-rhombicloop traveling-wave rectifying antenna for low power-density wireless power transmission applications," IEEE Trans. Microw. Theory Tech., vol. 51, no. 5, pp. 1548-1553, May 2003.

[8] — "Highly efficient C-band circularly polarized rectifying antenna array for wireless microwave power transmission," IEEE Trans. Antennas Propag., vol. 51, no. 6, pp. 1347-1356, Jun. 2004.

[9] W. H. Weedon, W. J. Payne, and G. M. Rebeiz, "MEMS-switched reconfigurable antennas," in Proc. IEEE AP-S Int. Symp., vol. 3, 2001, pp. 654-657.

[10] A. S. Daryoush and B. Choe, "Optically reconfigured active phased array antennas," in Proc. IEEE AP-S Int. Symp., vol. 1, 1988, pp. 44-47.

[11] B. C. C. Chang, Y. Qian, and T. Itoh, "A reconfigurable leaky mode/patch antenna controlled by PIN diode switches," in Proc. IEEE AP-S Int. Symp., vol. 4, 1999, pp. 2694-2697.

[12] M. N. Afsar, Y. Wang, and R. Cheung, "Analysis and measurement of a broad-band spiral antenna," IEEE Antennas Propag. Mag., vol. 46, no. 1, pp. 59-64, 2004.

[13] H. Morishita, H. Hamada, K. Nishida, and T. Nagao, "A wide-band circularly polarized dipole antenna," in Proc. IEEE AP-S Int. Symp., 1998, pp. $2348-2350$

[14] R. L. Li and V. Fusco, "Printed figure-of-eight wire antenna for circular polarization," IEEE Trans. Antennas Propag., vol. 50, no. 10, pp. 1487-1490, Oct. 2002.

[15] W. L. Stutzman and G. A. Thiele, Antenna Theory and Design. New York: Wiley, 1998, pp. 180-187.

[16] B. Edward and D. Rees, "A broad-band printed dipole with integrated balun," Microwave. J., pp. 339-344, May 1987.

[17] K. M. K. H. Leong, Y. Qing, and T. Itoh, "Surface wave enhanced broadband planar antenna for wireless applications," IEEE Microw. Wireless Compon. Lett., vol. 11, no. 2, pp. 62-64, Feb. 2001.

[18] C. Sabatier, "T-dipole arrays for mobile applications," IEEE Antennas Propag. Mag., vol. 45, no. 6, pp. 9-26, Dec. 2003.

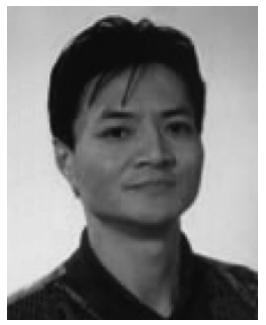

RongLin Li (M'02-SM'03) received the B.S. degree in electrical engineering from Xi' an Jiaotong University, China, in 1983 and the M.S. and Ph.D. degrees in electrical engineering from Chongqing University, China, in 1990 and 1994, respectively.

From 1983 to 1987, he was an Electrical Engineer with the Yunnan Electric Power Research Institute. From 1994 to 1996, he was a Postdoctoral Research Fellow with Zhejiang University, China. In 1997, he was with Hosei University, Japan, as a Hosei International Fund Research Fellow. Since 1998, he has been a Professor with Zhejiang University. In 1999, he was a Research Associate with the University of Utah. In 2000, he was a Research Fellow with Queen's University of Belfast, U.K. In 2001, he joined the ATHENA group as a Research Scientist with the Georgia Institute of Technology, Atlanta. His latest research interests include computational electromagnetics, modeling of antennas and microwave devices, and RF packaging design.

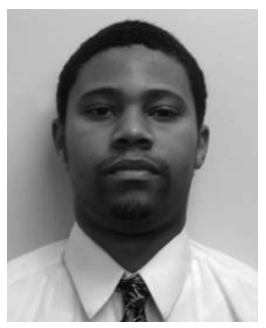

Gerald DeJean (S'03) received the B.Sc. degree in electrical engineering (with High Honors) from Michigan State University, East Lansing, in 2000. $\mathrm{He}$ is currently working toward the Ph.D. degree in electrical engineering at the Georgia Institute of Technology (Georgia Tech), Atlanta.

His current research interests include the design of compact antennas for integration into three-dimensional transceiver design, integration of antennas on multilayer substrates, computational electromagnetic modeling of antennas, and RF packaging and design.

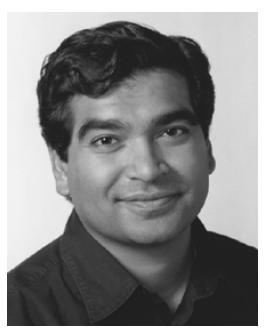

Joy Laskar (S'84-M'85-SM'02-F'05) received the B.S. degree in computer engineering (math/physics minors, highest honors) from Clemson University, Clemson, SC, in 1985 and the M.S. and Ph.D. degrees in electrical engineering from the University of Illinois, Urbana-Champaign, in 1989 and 1991, respectively.

Prior to joining the Georgia Institute of Technology (Georgia Tech), Atlanta, in 1995, he held faculty positions at the University of Illinois and the University of Hawaii. At Georgia Tech, he holds the Joseph M. Pettit Professorship of Electronics, is currently the Chair for the Electronic Design and Applications Technical Interest Group, the Director of Georgia's Electronic Design Center and the System Research Leader for the NSF Packaging Research Center. In 1998, he cofounded an advanced WLAN IC Company: RF Solutions, which is now part of Anadgics (Nasdaq: Anad). In 2001, he cofounded a next generation interconnect company: Quellan, which is developing collaborative signal processing solutions for enterprise applications. $\mathrm{He}$ also heads a research group with a focus on integration of high-frequency electronics with optoelectronics and integration of mixed technologies for next generation wireless and optoelectronic systems. His research has focused on high frequency IC design and their integration. He has authored or coauthored more than 200 papers, several book chapters (including three textbooks in development), numerous invited talks, and has 10 patents pending.

Dr. Laskar is a 1995 recipient of the Army Research Office's Young Investigator Award, a 1996 recipient of the National Science Foundation's CAREER Award, the 1997 NSF Packaging Research Center Faculty of the Year, the 1998 NSF Packaging Research Center Educator of the Year, the 1999 corecipient of the IEEE Rappaport Award (Best IEEE Electron Devices Society Journal Paper), the faculty advisor for the 2000 IEEE MTT IMS Best Student Paper award, 2001 Georgia Tech Faculty Graduate Student Mentor of the year, recipient of a 2002 IBM Faculty Award, the 2003 Clemson University College of Engineering Outstanding Young Alumni Award and the 2003 recipient of the Outstanding Young Engineer of the Microwave Theory and Techniques Society. He has been named the Joseph M. Pettit Professor of Electronics in the School of Electrical and Computer Engineering at Georgia Tech. For the 2004-2006 term, he has been appointed an IEEE Distinguished Microwave Lecturer for his seminar entitled "Recent Advances in High Performance Communication Modules and Circuits." 


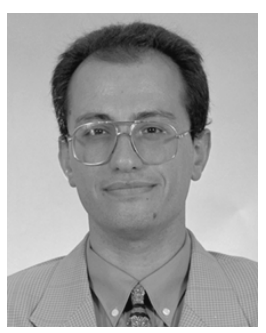

Manos M. Tentzeris (S'89-M'98-SM'03) received the diploma degree in electrical and computer engineering (magna cum laude) from the National Technical University of Athens, Greece, and the M.S. and Ph.D. degrees in electrical engineering and computer science from the University of Michigan, Ann Arbor.

He was a Visiting Professor with the Technical University of Munich, Germany for the summer 2002, where he introduced a course in the area of high-frequency packaging. He is currently an Associate Professor with the School of Electrical and Computer Engineering, Georgia Institute of Technology (Georgia Tech), Atlanta. He has helped develop academic programs in highly integrated/multilayer packaging for RF and wireless applications, microwave MEMs, SOP-integrated antennas and adaptive numerical electromagnetics (FDTD, multiresolution algorithms) and heads the ATHENA research group (15 researchers). He is the Georgia Tech NSF-Packaging Research Center Associate Director for RF Research and the RF Alliance Leader. He is also the leader of the RFID Research Group of the Georgia Electronic Design Center (GEDC) of the State of Georgia. He has given more than 40 invited talks in the same area to various universities and companies in Europe, Asia and America. He has published more than 180 papers in refereed journals and conference proceedings, eight book chapters, and he is in the process of writing three books.

Dr. Tentzeris is a member of the International Scientific Radio Union (URSI)Commission D, an Associate Member of EuMA, and a member of the Technical Chamber of Greece. He was the recipient of the 1997 Best Paper Award of the International Hybrid Microelectronics and Packaging Society for the development of design rules for low-crosstalk finite-ground embedded transmission lines. He received the 2000 NSF CAREER Award for his work on the development of MRTD technique that allows for the system-level simulation of RF integrated modules, the 2001 ACES Conference Best Paper Award, the 2002 International Conference on Microwave and Millimeter-Wave Technology Best Paper Award (Beijing, China) for his work on Compact/SOP-integrated RF components for low-cost high-performance wireless front-ends, the 2002 Georgia Tech-ECE Outstanding Junior Faculty Award, the 2003 NASA Godfrey "Art" Anzic Collaborative Distinguished Publication Award for his activities in the area of finite-ground low-loss low-crosstalk coplanar waveguides, the 2003 IBC International Educator of the Year Award, the 2003 IEEE CPMT Outstanding Young Engineer Award for his work on 3-D multilayer integrated RF modules, and the 2004 IEEE Transactions on Advanced Packaging Commendable Paper Award. He was also the 1999 Technical Program Co-Chair of the 54th ARFTG Conference, Atlanta, GA and he is the Vice-Chair of the RF Technical Committee (TC16) of the IEEE CPMT Society. He has organized various sessions and workshops on RF/Wireless Packaging and Integration in IEEE ECTC, IMS and APS Symposia in all of which he is a member of the Technical Program Committee in the area of "Components and RF." He is an Associate Editor of the IEEE TRANSACTIONS ON ADVANCED PACKAgING. 